\title{
Soutien en faveur de l'alimentation saine dans les écoles conformément à l'approche globale de la santé en milieu scolaire : évaluation au cours des premières années de la mise en ouvre de la Politique de l'Ontario concernant les aliments et les boissons dans les écoles
}

Taryn Orava, Ph. D. (1); Steve Manske, Ed. D. (2); Rhona Hanning, Ph. D. (1)

Cet article a fait l'objet d'une évaluation par les pairs.

Diffuser cet article sur Twitter

\section{Résumé}

Introduction : Les agences provinciales, nationales et internationales de santé publique reconnaissent l'importance des politiques en matière de nutrition en milieu scolaire, qui contribuent à instaurer des environnements sains respectant les recommandations en matière d'alimentation saine à l'intention des jeunes. Le soutien à l'échelle de l'école d'un mode de vie sain reposant sur les piliers de l'approche globale de la santé en milieu scolaire (milieux social et physique; enseignement et apprentissage; politique de santé en milieu scolaire; partenariats et services) a été positivement associé à des améliorations encourageantes des comportements des élèves en matière de santé. Notre étude a utilisé l'approche globale de la santé en milieu scolaire pour classifier, comparer et décrire le soutien à une alimentation saine lors de la mise en œuvre de la Politique de l'Ontario concernant les aliments et les boissons dans les écoles (N P/P 150).

\begin{abstract}
Méthodologie : Nous avons recueilli des données provenant d'écoles élémentaires et secondaires consentantes d'une zone peuplée de l'Ontario à deux reprises, en 2012-2013 (période I) et en 2014 (période II). Des représentants des écoles ont rempli l'enquête Planificateur des écoles en santé ainsi qu'un document d'analyse environnementale de l'alimentation, qui ont fait l'objet d'une attribution de scores et d'analyses de contenu. Le soutien de chaque école en faveur d'une alimentation saine a été classé comme relevant de la phase «lancement ", «prise de mesures » ou «maintenance » du continuum des écoles en santé pour les deux périodes, et comme étant " élevé/en hausse ", "modéré ", « faible/en baisse » entre la période I et la période II par rapport aux piliers de l'approche globale de la santé en milieu scolaire.
\end{abstract}

Résultats : Ont pris part à l'enquête 25 représentants scolaires (8 écoles élémentaires et 17 écoles secondaires). La plupart des écoles sont demeurées dans la catégorie « prise de mesures » $(\mathrm{n}=20)$ au cours des deux périodes, avec des niveaux divers de soutien aux piliers de la santé en milieu scolaire. Le milieu physique a fait l'objet du soutien le plus élevé (100\% de soutien " élevé/en hausse »), le milieu social a fait l'objet du soutien le plus faible (68\% de soutien «faible/en baisse »). Seules deux écoles ont atteint la plus haute note (maintenance) au cours de la période II. Les soutiens respectant la N P/P 150 semblent avoir été influencés par l'adhésion de l'administration, le soutien des intervenants et la pertinence du contexte local.

Conclusion : Une aide supplémentaire est nécessaire pour maintenir une alimentation saine dans les environnements alimentaires des écoles ontariennes.

Mots-clés : écoles, politique en matière de nutrition, santé scolaire, environnement alimentaire, santé globale en milieu scolaire
Points saillants

- D’après les résultats du Planificateur des écoles en santé, la plupart des écoles relèvent de la catégorie " prise de mesures » sur le continuum des écoles en santé. Cela suggère l'existence d'un soutien modéré en faveur de l'alimentation saine, avec une possibilité de progression.

- Les piliers du milieu physique et du milieu social ont été scindés afin d'examiner les caractéristiques de chaque type de milieu. Le milieu physique a fait l'objet d'un bon niveau de soutien, avec des espaces consacrés à l'alimentation des élèves sécuritaires et propres. Pour le pilier du milieu social, l'alimentation saine n'a pas souvent reçu de score élevé : peu d'écoles ont mentionné l'alimentation saine comme constituant une priorité « très élevée » (16\%) ou « élevée » $(8 \%)$.

- Les programmes et pratiques relevant des piliers de la formation et l'apprentissage, du partenariat et services et de la politique de santé en milieu scolaire nécessiteraient davantage d'adhésion de la part des administrateurs d'école, davantage de financement et une implication plus poussée de la part des intervenants des écoles.

Rattachement des auteurs :

1. École de santé publique et de systèmes de santé, Université de Waterloo, Waterloo (Ontario), Canada

2. Centre pour l'avancement de la santé des populations Propel, Waterloo (Ontario), Canada

Correspondance : Rhona Hanning, Université de Waterloo, Bureau BMH 3117, 200, avenue University Ouest, Waterloo (ON) N2L 3G1; tél. : 519-888-4567, poste 35685; téléc. : 519-746-6776; courriel : rhanning@uwaterloo.ca 


\section{Introduction}

Le milieu scolaire a les moyens d'encourager des comportements alimentaires positifs et sains chez les enfants dans leur jeune âge ${ }^{1-3}$. Comme la prévalence élevée d'obésité chez les enfants perdure, les écoles ont été incitées à adopter des politiques qui promeuvent officiellement les comportements alimentaires sains auprès de leurs élèves ${ }^{4-6}$. À l'échelle internationale, les politiques en matière de nutrition en milieu scolaire ont joué un rôle essentiel de soutien aux comportements alimentaires sains des enfants ${ }^{7-9}$. En 2011, s'inscrivant dans le sillage de la mise en œuvre de politiques en matière de nutrition en milieu scolaire dans plusieurs provinces et territoires du Canada, la province de l'Ontario a créé la Politique concernant les aliments et les boissons dans les écoles (note Politique/ Programme $n^{\circ} 150 \quad\left[\begin{array}{lll}N & P / P & 150\end{array}\right]$, un ensemble de normes nutritionnelles visant les aliments et boissons proposés à la vente dans les écoles, lors d'événements scolaires et par l'intermédiaire de programmes de nutrition ${ }^{10}$.

La politique précise que tous les lieux où l'on dispense de la nourriture dans les écoles doivent respecter la règle dite des 80-20 : parmi les aliments et boissons proposés à la vente, au moins $80 \%$ doivent appartenir à la catégorie " en vendre le plus ", au plus $20 \%$ doivent relever de la catégorie " en vendre moins " et $0 \%$ doivent correspondre à la catégorie "vente non autorisée $»^{10}$. Les catégories alimentaires de la N P/P 150 reposent sur les niveaux de matières grasses, de sucre, de sodium, de caféine et de calcium au sein de chaque catégorie d'aliment et de bois$\operatorname{son}^{10}$. Il incombe aux écoles de mettre en œuvre et d'assurer une surveillance constante de la règle des $80-20$, avec un maximum de dix jours d'exemption par année scolaire, durant lesquels même les aliments dont la vente n'est pas autorisée habituellement peuvent être proposés à la vente ${ }^{10}$. Des études préliminaires ont révélé que certains intervenants scolaires avaient rencontré des difficultés à mettre en œuvre la N P/P 150, ce qui a limité la capacité des écoles à la respecter pleinement ${ }^{11-13}$. Ces constatations vont dans le même sens que divers rapports qui indiquent que le respect des politiques en matière de nutrition prend généralement plusieurs années ${ }^{14,15}$.

Les politiques peuvent s'avérer défaillantes lorsque le contexte local ne favorise pas le changement (p. ex., la politique ne satisfait pas les besoins de la population cible, les personnes qui assument la responsabilité de la mise en œuvre n'apportent pas leur soutien au changement ou sont réticentes vis-à-vis de celui-ci) ${ }^{7-9}$. De plus, certains éléments indiquent que la mise en œuvre des politiques en matière de santé est plus efficace lorsqu'elle est combinée à d'autres approches encourageant les comportements sains chez les enfants ${ }^{16,17}$. L'approche globale de la santé en milieu scolaire, par exemple, a été alimentée par l'approche écologique et sociale en vertu de laquelle le fait de soutenir les comportements sains positifs des élèves, l'attention portée à l'environnement scolaire, la formation et l'apprentissage ainsi que les partenariats et les services sont les compléments indispensables à une politique ${ }^{7-9,18-23}$. De telles approches multidimensionnelles de la santé en milieu scolaire, également dénommées " écoles faisant la promotion de la santé » ou "santé coordonnée en milieu scolaire » visent à favoriser la santé et la réussite scolaire des élèves ${ }^{24}$. L'approche globale de la santé en milieu scolaire est un modèle d'analyse du milieu scolaire fondé sur quatre piliers interreliés, présentés dans le tableau 1. Il convient de remarquer que si le modèle combine le milieu social et le milieu physique, ceux-ci sont souvent observés et mesurés séparément.

Dans la mesure où la $\mathrm{N}$ P/P 150 a été créée sans stratégie de mise en œuvre globale correspondante, on ne sait pas si, ou comment, les piliers des milieux social et physique des écoles, de la formation et de l'apprentissage, de la politique en matière de santé en milieu scolaire et des partenariats et services sont en synergie en Ontario pour appuyer une alimentation saine. C'est pourquoi notre recherche, en utilisant l'approche globale de la santé en milieu scolaire comme guide, a visé à (1) classifier et comparer le niveau de soutien dont a fait l'objet l'alimentation saine dans l'approche globale en général et pour chacun des piliers de celle-ci lors de deux périodes, au cours des premières années de mise en œuvre de la N P/P 150; (2) repérer et décrire les caractéristiques du milieu scolaire pour lesquelles on a relevé de forts niveaux de soutien ou pour lesquels des améliorations ont été apportées aux piliers de la santé en milieu scolaire entre les périodes étudiées. Cette recherche a été l'occasion d'examiner l'évolution de la mise en œuvre d'une politique dans le contexte d'un soutien plus large à l'alimentation saine dans le cadre naturel des écoles d'une zone vaste et diversifiée de l’Ontario.

\section{Méthodologie}

\section{Cadre}

Notre recherche a été menée dans une zone peuplée de l'Ontario (Canada), en collaboration avec l'unité de santé publique régionale locale. L'identité de la région et des commissions scolaires régionales n'est pas divulguée dans cet article afin de préserver l'anonymat des écoles participantes. Les données ont été recueillies auprès de deux commissions scolaires (l'une publique, l'autre catholique) en deux temps : entre avril 2012 et juin 2013 (période I) et entre décembre 2013 et juin 2014 (période II). Ces périodes correspondent aux débuts de la mise en œuvre de la N P/P 150. Elles ont été choisies en fonction de l'environnement politique et des restrictions imposées sur le temps alloué au recrutement du personnel des écoles, conformément aux ententes d'examens déontologiques des commissions scolaires régionales. L'ensemble des éléments de cette recherche a été approuvé par le bureau d'éthique de la recherche de l'Université de Waterloo et par les comités d'examen scientifique des commissions scolaires participantes.

\section{Participants}

Nous avons recruté des écoles élémentaires et secondaires spécifiquement pour cette étude. Nous avons tiré un échantillon aléatoire de 38 écoles sur l'ensemble des écoles élémentaires $(\mathrm{N}=318)$ des commissions scolaires participantes. La randomisation a reposé sur la répartition géographique entre les trois municipalités concernées, le statut socioéconomique du voisinage des écoles, la taille de la population des écoles et une représentation égale entre les commissions scolaires publiques et catholiques. Bien que certaines écoles rurales figurent dans l'étude, la zone d'étude est majoritairement urbaine. Toutes les écoles secondaires de l'Ontario disposent de lieux de vente d'aliments sur place offrant un service quotidien, alors que les écoles élémentaires offrent des services plus limités (p. ex., des distributeurs automatiques) ou occasionnels (p. ex., des repas chauds mensuels de spécialité). Par conséquent, les écoles secondaires ont été considérées comme davantage susceptibles d'être touchées par les normes de la N P/P 150. 
TABLEAU 1

\section{Piliers de l'approche globale de la santé en milieu scolaire ${ }^{24,25}$}

\begin{tabular}{|c|c|}
\hline Piliers & Définition \\
\hline \multirow{2}{*}{$\begin{array}{l}\text { Milieu social } \\
\text { et physique }\end{array}$} & $\begin{array}{l}\text { Le milieu social comprend : } \\
\text { - la qualité des relations entre les membres du personnel, entre les élèves et entre } \\
\text { les membres du personnel et les élèves dans l'école; } \\
\text { - le bien-être émotionnel des élèves; } \\
\text { - les relations avec les familles et l'ensemble de la collectivité; } \\
\text { - l'aide à la communauté scolaire à faire des choix d'habitudes de vie saines en } \\
\text { favorisant l'acquisition de compétences et en encourageant l'autonomie et le } \\
\text { sentiment d'appartenance. }\end{array}$ \\
\hline & $\begin{array}{l}\text { Le milieu physique comprend : } \\
\text { - les bâtiments, les terrains, les aires de jeu et l'équipement qui se trouve à } \\
\text { l'intérieur et à l'extérieur de l'école; } \\
\text { - les commodités d'usage liées notamment à l'hygiène, à la pureté de l'air et à la } \\
\text { saine alimentation; } \\
\text { - les aires conçues pour favoriser le développement d'un sentiment de sécurité et } \\
\text { d'appartenance chez les élèves et prévenir les blessures; } \\
\text { - le milieu physique est sûr et accessible, et il favorise l'adoption de saines } \\
\text { habitudes de vie pour tous les membres de la communauté scolaire. }\end{array}$ \\
\hline \multirow[b]{2}{*}{$\begin{array}{l}\text { Enseignement } \\
\text { et apprentissage }\end{array}$} & $\begin{array}{l}\text { L'enseignement comprend : } \\
\text { - les programmes, ressources, et activités connexes formelles et informelles des } \\
\text { provinces ou territoires. }\end{array}$ \\
\hline & $\begin{array}{l}\text { L'apprentissage comprend : } \\
\text { - les connaissances, compréhensions et compétences pour que les élèves } \\
\text { améliorent leur santé et leur bien-être, ce qui fait progresser leurs résultats } \\
\text { d'apprentissage; } \\
\text { - des occasions de perfectionnement professionnel pour les membres du } \\
\text { personnel en santé et mieux-être. }\end{array}$ \\
\hline $\begin{array}{l}\text { Politique de } \\
\text { santé en milieu } \\
\text { scolaire }\end{array}$ & $\begin{array}{l}\text { Les politiques, lignes directrices et pratiques qui promeuvent et appuient le } \\
\text { mieux-être des élèves, ainsi que la réalisation et la formation d'un environnement } \\
\text { scolaire qui soit respectueux, accueillant et bienveillant pour l'ensemble des } \\
\text { membres de la communauté scolaire. }\end{array}$ \\
\hline \multirow[t]{2}{*}{$\begin{array}{l}\text { Partenariats et } \\
\text { services }\end{array}$} & $\begin{array}{l}\text { Les partenariats comprennent: } \\
\text { - les liens entre l'école et les familles des élèves; } \\
\text { - les relations de travail solidaires au sein des écoles (personnel et élèves), entre } \\
\text { les écoles, entre les écoles et d'autres organismes et groupes représentatifs dans } \\
\text { la communauté; } \\
\text { - la santé, l'éducation et d'autres secteurs collaborant pour faire progresser la } \\
\text { santé en milieu scolaire. }\end{array}$ \\
\hline & $\begin{array}{l}\text { Les services comprennent : } \\
\text { - les services en milieu scolaire et dans les collectivités destinés à soutenir et } \\
\text { promouvoir la santé et le mieux-être des élèves et du personnel scolaire. }\end{array}$ \\
\hline
\end{tabular}

Sources : Adapté du Consortium conjoint pancanadien pour les écoles en santé. Les quatre piliers de l'approche globale de la santé en milieu scolaire [en ligne]. Summerside (î.-P.-É.) : Consortium pancanadien pour les écoles en santé; 2017. En ligne à http://www.jcsh-cces.ca/index.php/a-propos/approche-globale-de-la-sante-en-milieu-scolaire/explications-des-quatre-piliers

Toutes les écoles secondaires de la région $(\mathrm{N}=62)$ ont été invitées à participer.

Les directeurs de chacune des écoles élémentaires et secondaires participantes ont été contactés par l'intermédiaire de lettres rédigées par les auteurs et diffusées par les infirmières en santé publique des écoles de la région. On a sélectionné pour chaque école un membre (qui a été soit autodésigné, soit choisi par l'administration scolaire) considéré comme compétent en matière d'initiatives liées à l'alimentation saine dans son école. Ce représentant a changé dans cinq cas entre la période I et la période II, du fait que cette recherche a eu lieu durant des années scolaires différentes.

\section{Instruments}

Les représentants des écoles participantes devaient remplir une version imprimée de l'enquête Planificateur des écoles en santé et aider un chercheur universitaire à remplir sur place une liste de vérification pour une analyse environnementale de l'alimentation en milieu scolaire.

\section{Enquête Planificateur des écoles en santé (PES)}

L'enquête PES a été conçue par le Consortium conjoint pancanadien pour les écoles en santé. Il s'agit d'un outil permettant de classifier le niveau de soutien d'une école envers une alimentation saine, l'activité physique, le contrôle du tabac et une santé mentale positive sur le continuum des écoles en santé (tableau 2), à partir de 9 à 12 questions fermées pour chacun des piliers de la santé en milieu scolaire ${ }^{25}$. L'approche globale de la santé en milieu scolaire classe les écoles dans les phases de " lancement ", de " prise de mesures " ou de " maintenance " en fonction du niveau de soutien envers le thème lié à la santé sélectionné ${ }^{26}$. Nous avons retenu le module d'alimentation saine pour notre étude, dans la mesure où il correspond directement aux piliers de l'approche globale de la santé en milieu scolaire. Les questions figurant dans ce module portent sur la présence et la fréquence de programmes liés à une alimentation saine (p. ex. la question suivante : "Votre école offre-t-elle des cours de cuisine, de jardinage, ou des visites des marchés fermiers locaux? ») et les pratiques liées à une alimentation saine (p. ex., "Votre école évite-t-elle ou non d'offrir des aliments malsains comme récompense à travers des politiques officielles, des pratiques informelles? »). Bien qu'aucune étude de validation ne soit disponible, le Conseil canadien de la santé et Agrément Canada ont reconnu que l'enquête PES était suffisamment fiable et valide à la suite des révisions de $2009^{27}$. Depuis le début de notre recherche, l'enquête PES a été reformatée et ses procédures de quotation ont été modifiées. Afin de renforcer la cohérence et la comparabilité des résultats, nous avons choisi d'utiliser la version imprimée de l'enquête PES avec le continuum des écoles en santé correspondant lors des deux périodes ${ }^{26}$. 
TABLEAU 2

\section{Le continuum des écoles en santé en $^{25}$}

\begin{tabular}{llll}
\hline \multicolumn{1}{|c}{ Lancement } & \multicolumn{1}{c}{ Prise de mesures } & \multicolumn{1}{c}{ Maintenance } \\
\hline $\begin{array}{l}\text { Ampleur des } \\
\text { recommandations de } \\
\text { la réunion }\end{array}$ & $\begin{array}{l}\text { En deçà des recomman- } \\
\text { dations de la réunion }\end{array}$ & $\begin{array}{l}\text { Satisfait certaines } \\
\text { recommandations, } \\
\text { mais pas toutes }\end{array}$ & $\begin{array}{l}\text { Satisfait les recomman- } \\
\text { dations ou va au-delà }\end{array}$ \\
\hline $\begin{array}{l}\text { Recommandations } \\
\text { pour l'avenir }\end{array}$ & $\begin{array}{l}\text { Très grande marge } \\
\text { de progression }\end{array}$ & $\begin{array}{l}\text { Marge de progression } \\
\text { possible }\end{array}$ & $\begin{array}{l}\text { Conserve le niveau } \\
\text { actuel d'engagement } \\
\text { en faveur d'une } \\
\text { alimentation saine } \\
\text { en milieu scolaire }\end{array}$ \\
\hline
\end{tabular}

Pour obtenir de plus amples renseignements au sujet du PES, veuillez consulter le site http://hsp.uwaterloo.ca.

\section{Liste de vérification de l'analyse contextuelle} de l'alimentation (ACA)

Nous avons élaboré une analyse contextuelle de l'alimentation (ACA) en plus du PES, avec 27 questions ouvertes et fermées sur l'état de la mise en œuvre de la $\mathrm{N} \mathrm{P} / \mathrm{P} 150$ et des programmes, pratiques et politiques liés à une alimentation saine au sein de chaque école. Au préalable, la liste de vérification a fait l'objet d'une mise à l'essai pilote dans des écoles secondaires situées dans une région différente ${ }^{28}$. La liste de vérification contient des questions comme : "Combien de jours d'exemption en vertu de la N P/P 150 votre école a-t-elle utilisés et dans quel but? » ou " Les élèves disposent-ils d'occasions de prendre part au jardinage? Dans l'affirmative, veuillez expliquer votre réponse. Dans la négative, quels obstacles ont empêché l'existence de telles occasions, et de quoi a-t-on besoin pour surmonter ces obstacles? " Nous avons modifié la liste de vérification de l'ACA pour la période II pour y intégrer des instructions destinées à faciliter les discussions conduites par le responsable du recueil de données et des questions supplémentaires pour noter les modifications apportées à l'environnement alimentaire en milieu scolaire depuis la période I (p. ex., " Le fournisseur de votre cafétéria est-il le même que l'année dernière? Si la réponse est négative, en quoi le nouveau fournisseur se distingue-t-il du précédent? ») (L'auteur-ressource peut fournir une copie de la liste de vérification de l'ACA sur demande).

\section{Attribution de scores et analyses}

Attribution de scores à l'enquête PES

Nous avons attribué des scores au PES de chaque école en utilisant une procédure en trois étapes et dont les résultats ont été comparés pour les deux périodes ${ }^{26}$.

\section{Étape 1 : Attribution de scores aux questions} relevant des piliers de la santé en milieu scolaire Les réponses aux questions relevant des piliers ont reçu la note de 1,0 (soit « lancement »), de 2,0 (" prise de mesures ») ou de 3,0 (« maintenance »).

\section{Étape 2 : Calcul de scores pour chaque pilier de la} santé en milieu scolaire

Nous avons calculé les scores moyens pour les questions correspondant aux piliers. Nous avons classé chaque pilier comme relevant de la catégorie "lancement ", " prise de mesures " ou "maintenance " sur la base des moyennes (c'est-à-dire, entre 1,0 et 1,99 pour " lancement ", entre 2,00 et 2,80 pour " prise de mesures » et entre 2,81 et 3,00 pour " maintenance »).

Étape 3 : Calcul d'un score global pour la santé en milieu scolaire

Nous avons calculé les moyennes pour les cinq scores des piliers de la santé en milieu scolaire et attribué un score global à la santé en milieu scolaire en fonction du continuum des écoles en santé (à savoir entre 1,0 et 1,99 pour " lancement ", entre 2,00 et 2,80 pour " prise de mesures » et entre 2,81 et 3,00 pour «maintenance »).

\section{Comparaison au fil du temps}

Nous avons comparé les scores globaux (c'est-à-dire les catégories lancement, prise de mesures ou maintenance) à la période I et à la période II, et les avons classés comme étant " faible/en baisse »; " modéré »; " élevé/en hausse » (tableau 3). Cette classification a été appliquée à chaque pilier de la santé en milieu scolaire.

\section{Analyse contextuelle de l'alimentation}

Nous avons obtenu des statistiques descriptives (moyenne et intervalles) en utilisant la version $23 \mathrm{du}$ logiciel statistique
SPSS (IBM, Armonk, NY, États-Unis). Les questions ouvertes (ACA) ont fait l'objet d'une analyse de contenu déductive au moyen de la version 10 du logiciel d'analyse qualitative NVivo (QSR International Pty Ltd., Melbourne, Australie). Les réponses ont été regroupées par pilier de la santé en milieu scolaire grâce à NVivo, et les résultats ont permis de décrire de façon plus approfondie les réponses ordinales aux questions de l'indicateur PES.

\section{Résultats}

\section{Échantillon de l'étude}

Parmi les 82 écoles invitées à participer, 45 ont répondu pour la période I ou la période II (taux de réponse de $55 \%$ ). Seules 25 écoles (8 écoles élémentaires et 17 écoles secondaires) ont rempli l'enquête PES pour les deux périodes et sont incluses dans nos résultats. L'enquête PES a été remplie par des enseignants (16 pour la période I et 16 pour la période II), des directeurs et de directeurs adjoints (11 pour la période I et 15 pour la période II), des responsables de programme et de service ( 3 pour la période I et 4 pour la période II), des membres du personnel de restauration (1 pour la période I et 1 pour la période II), un représentant de la commission scolaire (1 pour la période I) et des infirmières en santé publique (1 pour la période I et 1 pour la période II).

\section{Score global dans le continuum des écoles en santé pour la période I et la période II}

La figure 1 présente les scores globaux dans le continuum des écoles en santé pour la période I et pour la période II. Une majorité d'écoles se classe dans la phase de prises de mesure du continuum des écoles en santé pour la période I (6 écoles élémentaires et 14 écoles secondaires) et la période II (6 écoles élémentaires et 16 écoles secondaires). Très peu d'écoles ont obtenu la note la plus élevée, qui correspond à la phase de maintenance, pour la période I (1 école secondaire) ou II (2 écoles élémentaires).

\section{Comparaison des scores globaux de la période I et de la période II}

Comme l'illustre la figure 2, entre la période I et la période II, trois écoles ont progressé le long du continuum des écoles en santé (une école est passée de la catégorie de lancement à la catégorie de prise de 
TABLEAU 3

Classification du niveau de soutien des écoles à l'approche globale de la santé en milieu scolaire, période I (2012-2013) et période II (2014)

\begin{tabular}{|c|c|c|}
\hline $\begin{array}{c}\text { Changement du niveau de } \\
\text { soutien de la période I à la } \\
\text { période II }\end{array}$ & Description & $\begin{array}{l}\text { Notes en fonction du } \\
\text { continuum des écoles en santéa } \\
\text { (Période I à Période II) }\end{array}$ \\
\hline Soutien faible/en baisse & $\begin{array}{l}\text { Entre la période I et la } \\
\text { période II, les écoles ont régressé } \\
\text { sur le continuum des écoles en } \\
\text { santé ou sont demeurées à la } \\
\text { note la plus faible de la catégorie } \\
\text { "lancement». }\end{array}$ & $\begin{array}{l}\text { Maintenance } \rightarrow \text { Prise de mesures } \\
\text { Maintenance } \rightarrow \text { Lancement } \\
\text { Prise de mesures } \rightarrow \text { Lancement } \\
\text { Lancement } \rightarrow \text { Lancement }\end{array}$ \\
\hline Un soutien modéré est offert & $\begin{array}{l}\text { Entre la période I et la } \\
\text { période II, les écoles ont } \\
\text { maintenu une note correspon- } \\
\text { dant à la catégorie « prise de } \\
\text { mesures ». }\end{array}$ & $\begin{array}{l}\text { Prise de mesures } \rightarrow \text { Prise de } \\
\text { mesures }\end{array}$ \\
\hline Soutien élevé/en hausse & $\begin{array}{l}\text { Entre la période I et la } \\
\text { période II, les écoles ont progres- } \\
\text { sé sur le continuum des écoles } \\
\text { en santé ou sont demeurées à la } \\
\text { note la plus élevée de la } \\
\text { catégorie « maintenance ». }\end{array}$ & $\begin{array}{l}\text { Lancement } \rightarrow \text { Prise de mesures } \\
\text { Lancement } \rightarrow \text { Maintenance } \\
\text { Prise de mesures } \rightarrow \text { Maintenance } \\
\text { Maintenance } \rightarrow \text { Maintenance }\end{array}$ \\
\hline
\end{tabular}

${ }^{\text {a }}$ Voir le tableau 2.

mesures, et deux écoles sont passées de la catégorie de lancement à la catégorie de maintenance), 20 écoles sont demeurées dans la catégorie de la prise de mesures, une école dans la catégorie de lancement et une école a régressé (est passée de la catégorie de maintenance à la catégorie de prise de mesures). Le déplacement le long du continuum des écoles en santé s'est révélé dépendre des modifications en matière de niveau de soutien pour une alimentation saine dans les quatre piliers de la santé en milieu scolaire.

\section{Étendue de l'appui en matière}

d'alimentation saine fourni par le pilier de la santé en milieu scolaire

Le tableau 4 offre un aperçu du niveau de soutien en matière d'alimentation saine des écoles au sein de chaque pilier de la santé en milieu scolaire entre les périodes I et II. Les sections suivantes décrivent de quelle manière un soutien a été apporté en matière d'alimentation saine, en fonction des questions et réponses à l'indicateur du pilier de la santé globale en milieu scolaire de l'ACA.

FIGURE 1

Scores de soutien des écoles sur le continuum des écoles en santé entre la période I (2012-2013) et la période II (2014)

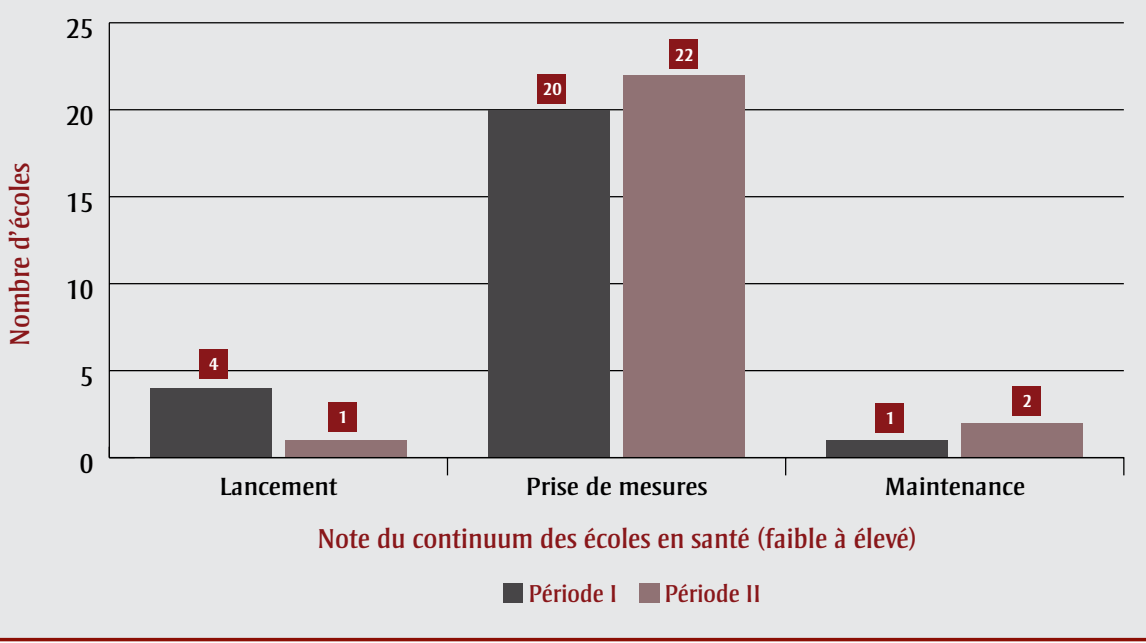

\section{Milieu social}

Six écoles (24\%) ont reçu une note générale " haute/en hausse " en matière de soutien au pilier du milieu social, et de nombreuses écoles se sont améliorées entre les deux périodes. Des indicateurs de l'appui à une alimentation saine (dérivés de l'enquête PES) ont été mentionnés par quelques écoles : une autoévaluation quant au fait que l'alimentation saine constitue une priorité élevée ( $8 \%$ des écoles) ou très élevée (16 \% des écoles) au sein de l'école, la présence d'un conseil sur la nutrition et l'alimentation des élèves au sein de l'école (40\%) ou l'organisation d'activités dans le cadre du mois de la nutrition (36 \%). Dans le but d'encourager les familles à adopter de saines habitudes alimentaires à la maison, les écoles distribuent le plus souvent des suggestions à propos des dîners et des collations santé (36\%) ou le Guide alimentaire canadien (16\%) aux parents et aux familles. De plus, afin de recueillir des commentaires sur les initiatives scolaires en matière d'alimentation saine auprès de la communauté scolaire dans son ensemble, les écoles ont déclaré avoir sollicité des suggestions auprès des élèves (40\%), des organisations de parents d'élève (24\%), des parents et des familles (16\%) et des membres du personnel (16\%).

\section{Milieu physique}

Le milieu physique est le pilier de l'approche santé en milieu scolaire qui a reçu le soutien le plus important. Toutes les écoles $(\mathrm{N}=25)$ pour les deux périodes ont signalé un niveau élevé de soutien du milieu physique en matière de saine alimentation (tableau 4). Les indicateurs du soutien du milieu physique sont le fait de disposer d'un nombre suffisant de chaises et de tables pour les repas des élèves, des fontaines d'eau accessibles et des lavabos pour permettre une hygiène des mains adéquate. De plus, les écoles élémentaires comme secondaires prévoient un temps suffisant pour prendre son dîner, socialiser avec ses amis et nettoyer (20 minutes à l'école élémentaire et entre 60 et 75 minutes à l'école secondaire).

Toutes les écoles élémentaires et secondaires ont fait la promotion d'une saine alimentation auprès des élèves, la plupart du temps par le biais d'affiches promotionnelles (p. ex., publicités pour les marchés fermiers, campagnes pour le mois de la nutrition) ou de panneaux dans la cafétéria des écoles secondaires (p. ex. cartes faisant la promotion des fruits de saison de la province, autocollants en faveur d'une 
FIGURE 2

Déplacement global des 25 écoles élémentaires et secondaires sur le continuum des écoles en santéa entre la période I (2012-2013) et la période II (2014)

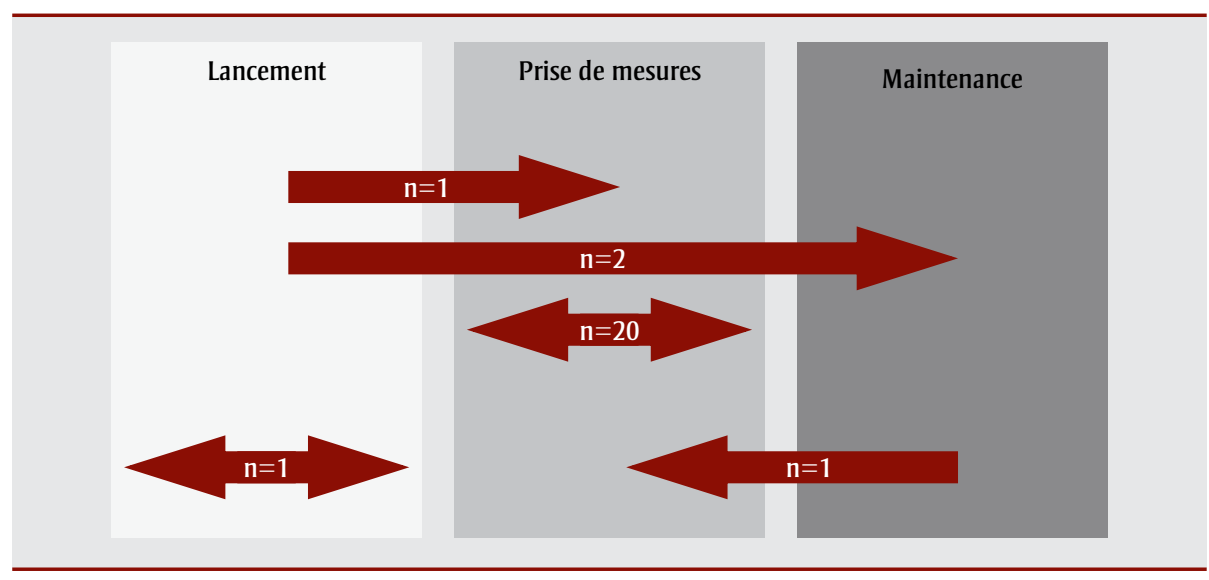

a Voir le tableau 2.

saine alimentation fournis par les autorités de santé publique régionales). La plupart des écoles secondaires $(\mathrm{n}=15)$ ont également utilisé des fresques et des dessins conçus par les élèves afin de sensibiliser aux choix alimentaires sains et d'en faire la promotion dans les cafétérias.

Toutes les écoles secondaires lors de la période II ( $\mathrm{n}=17)$ ont indiqué avoir organisé des activités pendant l'heure du dîner, par exemple un numéro intitulé « Fruit Ninja » pour sensibiliser aux avantages de la consommation de fruits et de légumes, des mini-concours de talents ou concours de chant, des réunions d'encouragement et des concours pour gagner des aliments compatibles avec la N P/P 150 (p. ex., " une minute pour gagner », « un rap pour un roulé ", " chanter pour une salade ", l'alimentation saine). Aucune activité de ce type n’a été signalée dans les écoles élémentaires, car les représentants ont déclaré que le temps du dîner était consacré à manger et à socialiser puis à une récréation à l'extérieur.

\section{Enseignement et apprentissage}

Le soutien apporté à l'alimentation saine en milieu scolaire au sein du pilier de l'enseignement et de l'apprentissage a été particulièrement fort pour la période II, grâce à des activités telles que l'apprentissage de compétences médiatiques sur des sujets spécifiques liés à la saine alimentation $(80 \%)$ et des sorties éducatives dans des marchés fermiers (40 \%) ou à l'épicerie locale (32\%). De plus, l'ACA a montré que $80 \%$ des écoles offraient des possibilités ainsi que des jeux-questionnaires sur

TABLEAU 4

Changement du niveau de soutien en faveur de l'alimentation saine entre la période I (2012-2013) et la période II (2014) pour 25 écoles élémentaires et secondaires, en fonction du pilier de santé en milieu scolaire

\begin{tabular}{|lccc}
\hline $\begin{array}{l}\text { Pilier de santé en } \\
\text { milieu scolaire }\end{array}$ & $\begin{array}{c}\text { Soutien faible/en baisse } \\
\text { entre la période I et la } \\
\text { période II } \\
\text { Nombre d'écoles }\end{array}$ & $\begin{array}{c}\text { Un soutien modéré est } \\
\text { offert entre la } \\
\text { période I et la } \\
\text { période II } \\
\text { Nombre d'écoles }\end{array}$ & $\begin{array}{c}\text { Soutien élevé/en } \\
\text { hausse entre la } \\
\text { période I et la } \\
\text { période II } \\
\text { Nombre d'écoles } \\
\text { n (\%) }\end{array}$ \\
\hline Milieu social & $\mathbf{n}(\%)$ & $2(8 \%)$ & $6(24 \%)$ \\
\hline $\begin{array}{l}\text { Milieu physique } \\
\text { Enseignement et } \\
\text { apprentissage }\end{array}$ & $17(68 \%)$ & - & $25(100 \%)$ \\
\hline $\begin{array}{l}\text { Politique de santé en } \\
\text { milieu scolaire }\end{array}$ & $7(4 \%)$ & $17(68 \%)$ & $7(28 \%)$ \\
\hline Partenariats et services & $7(28 \%)$ & $2(8 \%)$ & $16(64 \%)$ \\
\hline
\end{tabular}

de jardinage aux élèves dans des jardins sélectionnés, par exemple en entretenant un jardin commémoratif ou un jardin de la paix, en plantant un jardin d'herbes aromatiques ou en discutant de jardinage dans le club d'écologie de l'école. Le soutien apporté au développement des compétences alimentaires des élèves, présent dans de nombreuses écoles (60\%), a consisté en classes hôtelières et en une participation à des journées régionales d'éducation alimentaire ainsi qu'à des journées régionales dédiées aux collations.

De nombreuses écoles offraient des programmes réguliers de déjeuner (44\%, $\mathrm{n}=11$ ), de dîner ( $12 \%, \mathrm{n}=3$ ) et de collations $(8 \%, \mathrm{n}=2)$. La majorité des programmes de déjeuner ( $77 \%, \mathrm{n}=10$ ) était proposée aux élèves gratuitement en moyenne trois jours par semaine (entre 1 et 5 jours). Les programmes de dîner étaient offerts soit un, soit quatre, soit cinq jours par semaine, mais un programme de dîner « universel » (sans tenir compte de la capacité de payer) était proposé dans seulement l'une des trois écoles. Les programmes de collation $(n=2)$ étaient offerts gratuitement à tous les élèves, une ou trois fois par semaine. L'ensemble des programmes de nutrition ont été révisés par l'administration scolaire au moins une fois par an, et dans la mesure où ils sont offerts gratuitement, ils ne relèvent pas de la P/PM 150 ni de ses normes.

Lors de la période II, plusieurs représentants scolaires ont signalé ne pas avoir besoin de programme de déjeuner (32\%), de dîner (48 \%) ou de collation (52 \%). Les raisons avancées étaient l'absence de nécessité (c'est-à-dire que les élèves venaient toujours à l'école avec un dîner, ou que le quartier avait un statut socioéconomique élevé). À l'inverse, pour certaines écoles qui ne possédaient pas de programmes de nutrition pour les périodes I ou II, certains représentants ont indiqué qu'un programme de déjeuner (16\%), de dîner (32\%) ou de collation (12\%) serait souhaitable pour corriger, en particulier, les mauvaises habitudes alimentaires dont les enseignants ont été témoins dans les salles de classe et les cafétérias. Pour lancer un programme de ce type, les représentants ont déclaré avoir besoin d'un financement supplémentaire et de bénévoles.

\section{Politique d'écoles en santé}

Ce pilier a reçu du soutien de la part de nombreuses écoles (64\%, tableau 4) pour 
les deux périodes. Dans le but d'appuyer une saine alimentation à l'aide de mesures autres que la N P/P 150, les écoles ont veillé aux points suivants : des choix alimentaires sains disponibles à un prix raisonnable ou subventionné (24\% par le biais de politiques écrites, $32 \%$ par le biais de pratiques formelles), le bannissement des friandises sucrées comme récompenses dans les salles de classe ( $8 \%$ par le biais de politiques écrites, $48 \%$ par le biais de pratiques formelles) et une nourriture saine vendue lors d'activités de financement organisées à l'extérieur (16\% par le biais de politiques écrites, $12 \%$ par le biais de pratiques formelles). Les exemples d'activités de financement exemptes de nourriture étaient, d'après l'ACA, la vente de magazines, d'ustensiles de cuisine, de fleurs et de plantes, ainsi que des événements spéciaux comme des soirées cinéma, des soirées dansantes pour les élèves, des sorties de patinage et des activités de lavage de voitures.

Toutes les écoles élémentaires participantes disposaient d'une politique écrite empêchant les élèves de quitter l'école sans un mot écrit de leurs parents ou tuteurs, ce qui permet de restreindre l'accès aux magasins d'alimentation. À la fois pour les écoles élémentaires et secondaires, l'ACA a relevé le nombre de jours d'exemption utilisés en vertu de la N P/P 150. Aucune école n'a indiqué un nombre supérieur à la limite de 10 jours (moyenne $=3$; intervalle : entre 0 et 10 jours par an) lors des deux périodes. Les jours d'exemption ont servi à organiser des barbecues scolaires, des activités de financement à l'école (p. ex., vente de gâteaux, envoi de bonbons) et des événements liés au programme scolaire (p. ex., café français, concours d'entreprise commerciale).

\section{Partenariats et services}

Le pilier des partenariats et services a reçu des niveaux de soutien variables dans les écoles (tableau 4). Comme l'indiquent les données de la période II, les écoles ayant offert un niveau de soutien élevé ou en hausse en ce qui a trait au pilier des partenariats et services bénéficiaient souvent d'un personnel scolaire formé sur des sujets tels que la nutrition (44\%), l'enseignement d'un programme d'alimentation saine $(32 \%)$ et la promotion d'une image corporelle positive (28\%). Lors de la période I, lorsque la N P/P 150 a été prescrite pour la première fois, la majorité des écoles (88\%) ont envoyé un représentant de l'école afin qu'il reçoive la formation officielle offerte par le bureau de santé publique régional, en partenariat avec les commissions scolaires affiliées. Par ailleurs, de nombreuses écoles ont choisi de consulter au préalable les infirmières en santé publique présentes dans les écoles (68 \%) et le coordonnateur de la N P/P 150 rémunéré par la commission scolaire (56\%) afin de mettre en œuvre la Politique à l'échelle locale. Lors de la période II, seules $20 \%$ des écoles ont fait appel à une infirmière en santé publique, et le programme des coordonnateurs de la N P/P 150 financés par les commissions scolaires a été suspendu. Certaines écoles ont reçu des ressources liées à la N P/P 150 lors de la période II, qui ont été des ressources écrites (24\%) ou électroniques (20\%) relatives à la saine alimentation, à la nutrition ou à la politique.

Bien que la plupart des représentants scolaires ne se soient pas rencontrés pour discuter de la N P/P 150 avec une infirmière en santé publique lors de la période II, le bureau régional de santé publique a partagé des ressources et de l'information avec le personnel scolaire ( $88 \%$ ), et il a participé à l'élaboration et à la mise en œuvre de programmes (60\%) ou à la résolution de problèmes (32\%). Les écoles ont indiqué qu'elles mettaient en avant la promotion d'une alimentation saine au sein de la communauté élargie, ainsi que des activités pour les élèves avec les acteurs suivants : les organismes de santé communautaire (p. ex., la Fondation des maladies du cœur et de l'AVC, la Société canadienne du cancer, $32 \%$ ), la commission scolaire (28\%), le service municipal des parcs et des loisirs (24\%), un organisme destiné à la jeunesse (p. ex. YMCA, les clubs pour les garçons ou les filles, $16 \%$ ) et un club de remise en forme (12\%). Pour la période II, quatre écoles ont déclaré n’avoir eu aucun contact avec leur bureau régional de santé publique, et cinq autres écoles ont précisé ne pas disposer de liens communautaires externes pour soutenir une saine alimentation.

\section{Analyse}

Le fait de disposer de soutien au sein des quatre piliers de la santé en milieu scolaire au cours de la mise en œuvre de la Politique s'est révélé efficace pour favoriser des comportements sains de la part des élèves ${ }^{2,29}$. Notre étude a mis en lumière différents niveaux de soutien envers les piliers de la santé en milieu scolaire au cours de la mise en œuvre de la N P/P 150 au sein des écoles lors des deux périodes. Par exemple, toutes les écoles ont fait état d'un niveau de soutien élevé envers le pilier du milieu physique dans les deux périodes, en raison de l'engagement de l'Ontario à fournir des environnements scolaires sécuritaires et favorables du point de vue physique, conformément aux recommandations définies par l'OMS ${ }^{30}$. À l'inverse, le pilier du milieu social a reçu le score le plus faible, peutêtre en raison du fait que les activités parascolaires doivent être lancées par des personnes au sein du milieu scolaire.

Le directeur de l'école agit à titre de protecteur et a le pouvoir de faciliter ou de limiter l'adoption, la mise en œuvre et la durabilité des initiatives de la santé en

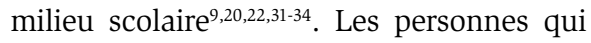
ont vraiment à cœur d'améliorer les comportements sains des élèves, appelées "champions de la santé», jouent le rôle d'intermédiaires clés qui favorisent en continu les initiatives de santé en milieu scolaire au sein de la salle de classe et en dehors de celle-ci $\mathrm{ci}^{20,21,35,36}$. Dans le cadre de notre étude, les champions de la santé en milieu scolaire étaient les enseignants, qui consacraient du temps en dehors de la salle de classe (pause dîner, après l'école) à mettre en œuvre des programmes et à superviser les élèves au sein de conseils d'action en matière de nutrition, de clubs d'écologie ou de concours extrascolaires portant sur les compétences alimentaires. Les réponses ouvertes fournies par les représentants scolaires indiquent un lien entre la présence des champions et les rares initiatives en faveur du pilier du milieu social.

Pour soutenir les champions, il est nécessaire d'offrir des heures rémunérées consacrées à la planification de programmes ou d'embaucher un coordonnateur externe pour favoriser le développement et la mise en œuvre des programmes avec les intervenants scolaires ${ }^{18,19,21-23}$. Lorsque les intervenants, qu'il s'agisse des enseignants, du personnel scolaire, des fournisseurs de services alimentaires, des familles, des partenaires au sein de la collectivité, des représentants chargés de la promotion de la santé ou des élèves eux-mêmes, sont mobilisés au cours du processus de prise de décision, on constate un niveau d'adhésion et un sentiment d'appartenance plus élevés, qui entraînent un engagement personnel en faveur de la mise en œuvre de l'initiative de santé en milieu 
scolaire ${ }^{19,22,23,37,38}$. Dans notre étude, les membres du personnel scolaire ont indiqué qu'ils n'étaient pas souvent consultés dans le cadre des discussions portant sur la saine alimentation au sein de l'école, et on pourrait imaginer que ce manque d'engagement ait contribué au faible soutien extrascolaire en matière d'alimentation saine dans le pilier du milieu social de l'approche globale de la santé en milieu scolaire. On doit travailler sur l'inclusion du personnel dans les discussions afin de s'assurer d'une adhésion en faveur de la promotion de la saine alimentation dans les écoles, ce qui permettra peut-être de favoriser l'adoption des normes de la N P/P 150.

Les écoles sollicitées dans notre étude ont fait état de différents niveaux de participation des intervenants. Les mesures telles que la mobilisation des membres de la collectivité au sein des discussions portant sur la saine alimentation, ainsi que le soutien apporté aux partenariats avec les organismes externes, ont été associés à des améliorations au sein des piliers de la santé en milieu scolaire et entre les périodes I et II. Par exemple, une école secondaire participante s'est associée à des organisations locales en matière de santé pour organiser une semaine d'événements liés au bienêtre, comprenant des cours de mise en forme physique, des cours de cuisine et des séminaires sur la promotion de la santé, dans le but d'appuyer la nouvelle $\mathrm{N} \mathrm{P} / \mathrm{P} 150$ et de renforcer les comportements sains en matière de santé. Des études ont montré que les partenariats réussis permettaient d'augmenter la disponibilité des soutiens, des ressources et des possibilités de promotion des messages concernant une saine alimentation dans les espaces publics, en dehors de l'école, où les enfants vivent, apportent parfois peu d'avantages aux écoles ${ }^{9,18,40}$. Cela pourrait expliquer pourquoi les écoles de notre étude ont fait état de faibles niveaux de soutien à l'appui du pilier des partenariats et services : cinq écoles n'ont tissé aucun lien avec des partenaires externes, et quatre écoles n'ont pas communiqué avec l'infirmière en santé publique de leur école au cours de l'année scolaire précédente. La littérature indique que certains représentants scolaires éviteraient de rechercher la participation de groupes d'intervenants parce qu'il « est plus rapide de prendre seul des décisions plutôt que de collaborer $»^{41, p .524}$.

Parfois, lorsqu'ils sont consultés, les intervenants offrent des résistances. Par exemple, certains employés des services alimentaires envisagent les stratégies de saine alimentation d'un point de vue commercial plutôt que du point de vue de la promotion de la santé, ou les parents peuvent être en désaccord avec les messages de promotion de la santé ou ne pas être en mesure d'appliquer ces principes à la maison en raison de problèmes tels que l'insécurité alimentaire, les normes culturelles ou les croyances en matière de santé personnelle $e^{20,38}$. Des recherches qualitatives sont à mener pour mieux comprendre les obstacles à la création et au maintien de partenariats au sein de la collectivité dans le but d'appuyer les milieux scolaires offrant une alimentation saine.

Le manque de financement constitue un autre frein à la mise en œuvre de l'approche globale de la santé en milieu scolaire. Par le passé, lorsque les écoles ont reçu un financement externe, les représentants ont été en mesure de favoriser l'approche globale de la santé en milieu scolaire en fonction des priorités de l'environnement scolaire ${ }^{9,19,20}$. Inversement, en l'absence de financement supplémentaire, les membres du personnel scolaire ont indiqué ne pas être en mesure de participer aux initiatives de l'approche globale de la santé en milieu scolaire car ils n'étaient pas rémunérés pour ces efforts ${ }^{20,21,38,39}$. Nous avons déterminé que la disponibilité en financement provenant de subventions gouvernementales, d'activités de financement externes ou de frais versés par les élèves a permis à certaines écoles d'offrir de manière régulière des programmes gratuits ou subventionnés de déjeuner, de dîner ou de collation. Dans certains cas cependant, les écoles qui souhaitaient mettre en place des programmes de nutrition pour les élèves n'ont pas pu le faire en raison d'un manque de financement.

Lorsqu'un financement provenant de sources non gouvernementales externes était disponible pour une école (c'étaient les seules sources de financement disponibles dans le cadre de notre étude), les élèves ont pris part à des activités de développement des compétences, comme le jardinage ou la cuisine, puisqu'il était possible de se procurer les outils et le matériel nécessaires (plantes, ustensiles de cuisine, ingrédients). Deux écoles élémentaires de notre étude ont obtenu un financement et de la nourriture de la part d'une épicerie locale afin d'organiser des séances d'éducation à la nutrition pour les élèves et les parents. Elles ont également obtenu le soutien des infirmières en santé publique des écoles formées à la promotion d'une saine alimentation et ont participé aux demandes de subventions. Des efforts supplémentaires sont requis pour sensibiliser les représentants scolaires aux possibilités de financement susceptibles de répondre aux besoins spécifiques des environnements scolaires.

Le contexte joue un rôle important pour une mise en œuvre réussie des initiatives l'approche globale de la santé en milieu scolaire. Lorsque les stratégies de mise en œuvre tiennent compte de ce contexte, les écoles sont en mesure d'harmoniser leurs priorités avec la politique de santé en milieu scolaire s, $18,20,22,41,42$. Par exemple, le projet Alberta Project Promoting active Living and healthy Eating in Schools (écoles APPLE) vise à intégrer le bien-être dans la culture scolaire par le biais d'événements et d'activités suivies et en incluant des enseignements sur le bien-être dans le programme scolaire ${ }^{43}$. Grâce à un financement, chaque école a été en mesure de prévoir des périodes pour que le personnel communique avec un intermédiaire de santé scolaire et donne la priorité aux politiques et pratiques promouvant le bien-être à l'école ${ }^{22}$. Une fois que les directeurs d'école ont saisi la philosophie du projet et sa conformité aux priorités de l'école, il a été possible de mettre en œuvre avec succès les initiatives de l'approche globale de la santé en milieu scolaire dans les écoles APPLE et d'en assurer le suivi ${ }^{22}$. Les données recueillies dans le cadre de notre étude en matière de contexte dynamique des écoles soulignent qu'il est nécessaire que de multiples représentants occupant des postes d'autorité (ministère de l'Éducation, commission scolaire, direction d'école, champions en milieu scolaire) collaborent avec les intervenants scolaires pour élaborer des stratégies de soutien durable à une saine alimentation dans toutes les dimensions de l'approche globale de la santé en milieu scolaire.

\section{Points forts et limites}

À notre connaissance, cette étude constitue l'une des premières à évaluer les piliers de l'approche globale de la santé en milieu scolaire depuis la mise en œuvre de la $\mathrm{N} \mathrm{P} / \mathrm{P} 150$, ce qui permet de mieux comprendre la nature de l'appui des écoles ontariennes en matière d'alimentation saine. Des recherches plus poussées permettraient de tirer parti des résultats 
actuels afin d'examiner la relation doseréponse entre la présence de soutien en matière d'alimentation saine et les résultats liés à la santé au niveau de la population au sein de chaque école et entre celles-ci.

Cette étude comporte cependant des limites. Le taux de réponse relativement faible peut être attribué aux différentes priorités concurrentes au sein des écoles ou à la grève du zèle des enseignants au cours de la période I (2012-2013) de notre étude, ce qui a limité les communications entre les chercheurs et les administrateurs scolaires. Dans cinq cas, les représentants scolaires ont changé entre les deux périodes, ce qui a offert des perspectives différentes pour les périodes de collecte de données. De plus, il pourrait y avoir une partialité liée à l'autoévaluation : les représentants qui participaient à la promotion des pratiques alimentaires saines à l'école peuvent avoir rapporté des résultats qui diffèrent de ceux des personnes moins mobilisées. Par ailleurs, les résultats de cette étude donnent seulement un aperçu des environnements de saine alimentation dans les écoles, car aucune mesure n'a été renouvelée au cours de l'année scolaire. Toutes les écoles invitées n'ayant pas participé à l'étude, une éventuelle partialité des bénévoles est à envisager.

Enfin, l'approche globale de la santé en milieu scolaire ne relève que de l'environnement scolaire et ne prend pas en compte l'environnement externe, tels qu'une grève du zèle, la quantité et le type d'aliments concurrentiels extérieurs à l'école, la mise en évidence par les médias de l'importance d'une saine alimentation et les autres facteurs environnementaux à la maison ou dans les centres de loisirs susceptibles d'avoir une incidence sur les comportements des élèves en matière de saine alimentation.

\section{Conclusion}

La Politique constitue un élément essentiel des initiatives e l'approche globale de la santé en milieu scolaire, car elle fournit un appui du sommet vers la base et le renforcement durable nécessaire pour encourager les personnes à changer de comportement $^{7,8}$. Lorsqu'elles sont prescrites par un organisme faisant autorité, les politiques en matière de santé peuvent également aider les milieux scolaires à établir des normes et des priorités pour les autres initiatives de l'approche globale de la santé en milieu scolaire ${ }^{43,44}$. Cela nécessite des approches de la base vers le sommet supplémentaires, exhaustives et intégrées pour motiver personnellement les acteurs du changement (les directeurs d'école, les enseignants, le personnel, les membres de la collectivité, les parents, les élèves) à adopter et à conserver les priorités de l'approche globale de la santé en milieu scolaire ${ }^{45,46}$. Ces stratégies doivent être cohérentes et orchestrées, avec une stratégie de mise en œuvre qui cible simultanément plusieurs dimensions de la santé des élèves et de l'environnement alimentaire $^{47}$. Sans une aide structurée tout au long du processus de mise en œuvre, les initiatives de l'approche globale de la santé en milieu scolaire vacilleront ${ }^{23}$. Les résultats des méta-analyses ont démontré que des stratégies de mise en œuvre efficaces permettent d'obtenir de meilleurs résultats $^{46}$. Par conséquent, les écoles doivent mettre au point un plan de mise en œuvre structuré en faveur de la politique de santé en milieu scolaire, qui prenne en compte les priorités spécifiques à l'école (milieu social), sa structure physique (milieu physique), le respect du programme scolaire et l'atteinte des objectifs de réussite scolaire (enseignement et apprentissage), le soutien aux politiques et pratiques informelles existantes (politique d'écoles en santé) et les appuis durables et les ressources qui peuvent être obtenus par l'école et les partenaires communautaires (partenariats et services).

Il existe actuellement une réelle opportunité à mettre en pratique les leçons apprises grâce à la recherche, car le ministère de l'Éducation ontarien cherche à mobiliser les membres du milieu scolaire dans le cadre de la Stratégie ontarienne pour le bien-être en milieu scolaire ${ }^{48}$. Cette initiative vise à mieux comprendre le bien-être physique, cognitif, émotionnel et social des enfants, et elle s'appuie sur le système existant pour soutenir toutes les dimensions du développement et de la santé des enfants. Nous recommandons que le ministère de l'Éducation ontarien, les partenaires communautaires et les représentants scolaires envisagent d'utiliser l'approche globale de la santé en milieu scolaire pour mieux comprendre comment le bien-être, en particulier les comportements alimentaires des élèves, est influencé par toutes les dimensions de l'environnement scolaire. Par ailleurs, des outils comme le Planificateur des écoles en santé peuvent aider le ministère et les communautés scolaires à découvrir les activités qui fonctionnent bien et celles qui nécessitent un soutien supplémentaire.

\section{Remerciements}

Ce manuscrit contient des données provenant du Planificateur des écoles en santé, un outil élaboré par le Consortium conjoint pancanadien pour les écoles en santé en partenariat avec le Centre pour l'avancement de la santé des populations Propel de l'Université de Waterloo. La recherche sur le PES a été soutenue financièrement par la subvention $\mathrm{n}^{\circ}$ 2011-701019 de la Société canadienne du cancer. Les auteurs ont reçu le soutien de la subvention des Instituts de recherche en santé du Canada en intervention auprès des populations pour la prévention des maladies chroniques et des services de santé publique de la région de Peel.

\section{Conflits d'intérêts}

Les auteurs déclarent n'avoir aucun conflit d'intérêts.

\section{Contribution des auteurs}

TO a contribué à tous les aspects de la recherche : le modèle de recherche, l'élaboration des instruments, la collecte des données, l'analyse, la rédaction du manuscrit et l'approbation de la version finale. $\mathrm{RC}$ et SM ont contribué au modèle de recherche, à l'élaboration des outils, à la supervision de la collecte et de l'analyse des données ainsi qu'à l'approbation du manuscrit définitif.

\section{Références}

1. Aldinger CE et JT Jones. Healthy nutrition: an essential element of a healthpromoting school [Internet]. Genève (CH) : Organisation mondiale de la santé; 1998 [consultation en 2014]. [Série d'information sur la santé dans les écoles de l'OMS : document $\left.n^{\circ} 4\right]$. En ligne à : http://www.who.int/school _youth_health/media/en/428.pdf?ua = 1

2. Diressen CE, AJ Cameron, LE Thornton, SK Lai et LM Barnett. Effect of changes to the school food environment on eating behaviours and/or body weight in children: a systematic review. Obes Rev. 2014;15(12):968-982.

3. Story M, KM Kaphingst et S French. The role of schools in obesity prevention. Future Child. 2006;16:109-142. 
4. Comité d'experts pour la santé des enfants. L'urgence d'agir : stratégie pour des enfants en santé. Toronto (Ont.) : Imprimeur de la Reine pour l'Ontario; [2013]. [Catalogue $\left.n^{\circ} 017309\right]$. En ligne à : http://www.health.gov.on .ca/fr/common/ministry/publications /reports/healthy_kids/healthy_kids.pdf

5. Agence de la santé publique du Canada. Environnements favorables à l'apprentissage : la saine alimentation et l'activité physique dans l'approche globale de la santé en milieu scolaire. Revue canadienne de santé publique. 2010;101 (Supplément 2):S1-S34.

6. Organisation mondiale de la Santé (OMS). Stratégie mondiale pour l'alimentation, l'exercice physique et la santé : Surpoids et obésité de l'enfant [Internet]. Genève (CH) : OMS; [2014]. En ligne à : http://www.who.int /dietphysicalactivity/childhood/fr/

7. Ardzejweska K, R Tadros et D Baxter. A descriptive study on the barriers and facilitators to implementation of the NSW (Australie) Healthy School Canteen Strategy. Health Educ J. 2012; 72(2):136-45.

8. Gleddie DL. The devil is in the details: development of policy and procedure in the Battle River Project. Health Educ J. 2010;71(1):30-38.

9. Inchley J, J Muldoon et C Currie. Becoming a health promoting school: evaluating the process of effective implementation in Scotland. Health Promot Int. 2007;22(1):65-71.

10. Ministère de l'Éducation de l'Ontario. Politique/Programmes Note no 150. Politique concernant les aliments et les boissons dans les écoles [Internet]. Toronto (Ont.) : Imprimeur de la Reine pour l'Ontario; [mise à jour le 4 octobre 2010]. En ligne à : http://www.edu .gov.on.ca/extra/fre/ppm/150.html

11. Chaleunsouk L et B Kutsyuruba. Ontario schools' readiness for School Food and Beverage Policy implementation. J Educ Policies Curr Pract. 2014; $1(1): 13-29$.

12. Vine M et SJ Elliott. Examining locallevel factors shaping school nutrition policy implementation in Ontario, Canada. Public Health Nutr. 2014;17(6): 1290-1298.
13. Vine MM, SJ Elliott et KD Raine. Exploring implementation of the Ontario School Food and Beverage Policy at the secondary-school level: a qualitative study. Can J Diet Pract Res. 2014;75(3):118-124. doi: 10.3148/cjdpr -2014-003.

14. Rideout K, R Levy-Milne, C Martin et AS Ostry. Food sales outlets, food availability, and the extent of nutrition policy implementation in schools in British Columbia. Can J Public Health. 2007;98(4):246-250.

15. Samuels SE, KS Hutchinson, L Craypo, J Barry et SL Bullock. Implementation of California state school competitive food and beverage standards. J Sch Health. 2010;80(12):581-587.

16. Fung C, S Kuhle, C Lu et coll. From "best practice" to "next practice": the effectiveness of school-based health promotion in improving healthy eating and physical activity and preventing childhood obesity. Int $\mathrm{J}$ Behav Nutr Phys Act [Internet]. 2012;9:27. En ligne à : http://dx.doi.org/10.1186/1479 $-5868-9-27$

17. Fung C, JLD McIsaac, S Kuhle, SFL Kirk et PJ Veugelers. The impact of a population-level school food and nutrition policy on dietary intake and body weights of Canadian children. Prev Med. 2013;57(6):934-940.

18. Deschesnes M, C Martin et A Jomphe Hill. Comprehensive approaches to school health promotion: how to achieve broader implementation? Health Promot Int. 2003;18(4):387-396.

19. Gleddie DL et EP Hobin. The Battle River Project: school division implementation of the health-promoting schools approach: assessment for learning: using student health and school capacity measures to inform action and direct policy in a local school district. Glob Health Promot. 2011;18(1):39-42.

20. McIsaac JLD, K Read, PJ Veugelers et SFL Kirk. Culture matters: a case of school health promotion in Canada. Health Promot Int. 2017;32(2):207-217. doi:10.1093/heapro/dat055.
21. Rasberry CN, S Slade, DK Lohrmann et RF Valois. Lessons learned from the whole child and coordinated school health approaches. J Sch Health. 2015; 85(11):759-765.

22. Roberts E, N McLeod, G Montemurro, PJ Veugelers, D Gleddie et KE Storey. Implementing comprehensive school health in Alberta: the principal's role. Health Promot Int. 2016;31(4):915-924. doi: 10.1093/heapro/dav083.

23. Senior E. Becoming a health promotion school: key components of planning. Glob Health Promot. 2012;19(1):23-31.

24. Veugelers PJ et ME Schwartz. Approche globale de la santé en milieu scolaire au Canada. Revue canadienne de santé publique. 2010;101 (Supplément 2):S5-S8.

25. Consortium conjoint pancanadien pour les écoles en santé. Approche globale de la santé en milieu scolaire [en ligne]. Summerside (Î.-P.-É.) : Consortium pancanadien pour les écoles en santé; 2015. En ligne à : http://www.jcsh -cces.ca/index.php/a-propos/approche -globale-de-la-sante-en-milieu-scolaire

26. Healthy School Planner Survey. Background information from the Joint Consortium for School Health Survey. Waterloo (Ont.) : Pan-Canadian Joint Consortium for School Health; 2013. En ligne à : http://hsp.uwaterloo.ca/

27. Agrément Canada. Planificateur des écoles en santé du Consortium conjoint pancanadien pour les écoles en santé (CCES) [Internet]. Ottawa (Ont.) : Agrément Canada; 2017. En ligne à : https://accreditation.ca/fr/node/5903

28. Habayeb D. Evaluating implementation of the Ontario School Food and Beverage Policy (P/PM 150) in Waterloo region: role of food services (mémoire de maîtrise). Waterloo (Ont) : Université de Waterloo; 2013.

29. Langford R, CP Bonnell, HE Jones, et coll. The WHO Health Promoting School framework for improving the health and well-being of students and their academic achievement. Cochrane Database Syst Rev. 2014;(4):CD008958. doi: 10.1002/14651858.CD008958.pub2. 
30. Wargo J. The physical school environment: an essential component of a health-promoting school. Genève $(\mathrm{CH})$ : Organisation mondiale de la santé; 2004. [Série d'information sur la santé dans les écoles de l'OMS : document no 2].

31. Fullan MG. Successful school improvement: the implementation perspective and beyond. Philadelphia (PA): Open University Press; 1992.

32. Rohrbach LA, JW Graham et WB Hansen. Diffusion of a school-based substance abuse prevention program: predictors of program implementation. Prev Med. 1993;22(2):237-260.

33. Storey KE, H Spitters, C Cunningham, M Schwartz et PJ Veugelers. Implementing comprehensive school health: teachers' perceptions of the Alberta Project Promoting active Living and healthy Eating in Schools - APPLE schools. PHEnex J [Internet]. 2011; $3(2): 1-18$. En ligne à : http://ojs .acadiau.ca/index.php/phenex/article /view/1422

34. Storey KE. Implementing comprehensive school health: from 'doing' to 'facilitating.' Présenté à : Ever Active Schools, 4th annual Shaping the Future Conference; 24 au 26 janvier 2013; Kananaskis, (Alberta), Canada.

35. Stolp S, E Wilkins et KD Raine. Developing and sustaining a healthy school community: essential elements identified by school health champions. Health Educ J. 2014;74(3):299-311.

36. Valois RF, TL Lewallen, S Slade et A Tasco. The ASCD healthy school communities projects: formative evaluation results. Health Educ. 2015;115(3/4): 269-284.

37. O’Hara J et G McNamara. Process and product issues in the evaluation of school development planning. Evaluation. 2001;7(1):99-109.

38. Lucarelli JF, K Alaimo, E Mang et al. Facilitators to promoting health in schools: is school health climate the key? J Sch Health. 2014;84(2):133140.
39. Holosko MJ et JM Dunlop. Evaluating interorganizational approaches to service delivery: a case example of the family violence serve project in Kent county, Ontario. Can J Program Eval. 1992;7(2):115-129.

40. Marshall BJ, MM Sheehan, Northfield JR, S Maher, R Carlisle et LH St. Leger. School-based health promotion across Australia. J Sch Health. 2000;70(6): 251-252.

41. Rowling L. The adaptability of the health promoting schools concept: a case from Australia. Health Edu Res. 1996;11(4):519-526.

42. St. Leger L. Schools, health literacy and public health: possibilities and challenges. Health Promot Int. 2001; 16(2):197-205.

43. Sabatier PA. Top-down and bottom-up approaches to implementation research. Dans : Hill M, editor. The policy process: a reader. 2e édition. New York (NY): Harvester Wheatsheaf; 1997: 267-293.

44. Tang KC, D Nutbeam, C Aldinger et coll. Schools for health, education and development: a call for action. Health Promot Int. 2009;24(1):68-77.

45. Gleddie DL et NE Melnychuck. An introduction to the Battle River Project: district implementation of a health promoting schools approach. Phys Health Educ J. 2009;75(2):24-31.

46. Durlak JA et EP DuPre. Implementation matters: a review of research on the influence of implementation on program outcomes and the factors affecting implementation. Am J Community Psychol. 2008;41(3-4): 327-350.

47. Allensworth DD et LJ Kolbe. The comprehensive school health program: exploring an expanded concept. J Sch Health. 1987;57(10):409-412.

48. Ministère de l'Éducation de l'Ontario. Promouvoir le bien-être dans le système d'éducation de l'Ontario [Internet]. [4 mai 2016; mise à jour 3 nov. 2016] En ligne à : http://www.edu.gov.on .ca/fre/about/wellbeing2.html 